\title{
Straightforward routes from CIFs to 3D printed crystallographic models
}

Peter Moeck $^{1}$, Paul DeStafano ${ }^{1}$, Izzy Cheung ${ }^{1}$, Werner Kaminsky ${ }^{2}$, Trevor Snyder ${ }^{3}$

${ }^{1}$ Portland State University, Portland, United States, ${ }^{2}$ Department of Chemistry, University of Washington, Seattle, United States, ${ }^{3} 3 \mathrm{D}$ Systems Corporation, Wilsonville, United States

E-mail: pmoeck@pdx.edu

Crystallographic models of molecules, Bravais lattices, crystal structures, space and point group symmetries, crystal defects, and crystal morphologies are all examples of what can be encoded in the well documented and versatile Crystallographic Information Framework (CIF) file format that is utilized by the open-access databases mentioned below. The Crystallography Open Database (COD, www.crystallography.net) has accumulated more than 370,000 entries and is the world's premier open-access source for the CIFs of structures of small molecules and small to medium sized unit cell crystals. There are currently four mirrors of this database worldwide (http://cod.ibt.lt/, http://cod.ensicaen.fr/, http://qiserver.ugr.es/cod/, and http://nanocrystallography.org/). Other open access sources of CIFs are a related Open Access Crystallography project at Portland State University (nanocrystallography.research.pdx.edu) and the Worldwide Protein Data Bank (http://www.wwpdb.org/). While 3D printing has already been available for some 20 years, cost and performance improvements have only recently made 3D printing practical for usage by researchers and educators [1-3]. The 3D printing process requires files of the models in the industry-wide accepted Standard Tessellation Language (STL, *.stl) or Virtual Reality Modeling Language (VRML, *.wrl) syntax. While STL files only allow for monochrome 3D printing, multiple color printing is enabled with WRL files. A significant recent innovation by the 3D printing industry is water soluble support material that is to be removed after the 3D printing process. Virtual reality computer programs (http://cad4.cpac.washington.edu) that include direct conversions from CIF to STL and WRL were written by Werner Kaminsky and are freely available for non-commercial usages. One of these programs allows for the creation of crystal morphology models form CIFs. The Nano-Crystallography group at Portland State University intends to upgrade both its mirror of the COD (i.e. nanocrystallography.org) and its Open Access Crystallography project websites with on-line 3D print file creating capabilities. Code from the searchable 3D Print and Crystal Symmetry Explorer website (https://chemapps.stolaf.edu/jmol/3dprint/) [3] is to be incorporated into our sites for this purpose. Bob Hanson and coworkers utilize JSmol scripting (i.e. the non-Java HTML5 version of Jmol) on their site to enable not only the interactive display of the structural information in CIFs with a multitude of options (via the Crystal Symmetry Explorer), but also the interactive design of models of the structures that are to be printed in 3D [3]. The 3D print files of the models that the user created are saved in the file formats WRL, STL, or X3D (which is the Extensible Markup Language version of VRML) directly to the local machine on which the user works. Of particular interest to crystallography educators will probably be 3D printed models consisting of blocks of multiple unit cells. Creating these bocks is just one of many design options of the above mentioned route [3]. A selection of ready made 3D print files of crystallographic models with educational relevance is provided at nanocrystallography.research.pdx.edu/3d-print-files/ for free downloads.

[1] Moeck, P. et al. (2014). J. Mater. Edu. 36, 77-96. [2] Stone-Sundberg, J. et al. (2015). Cryst. Res. Technol. 50, $432-44$. [3] Scalfani, V. F. et al. (2016). Cheminform 8, 66

Keywords: $3 \mathrm{D}$ print files, CIF, open-access crystallographic databases 